\title{
A Teleodontologia nos cursos da cidade de São Paulo
}

\author{
Teledentistry in graduation courses in the city of São Paulo \\ Teledontología en cursos en la ciudad de São Paulo
}

Recebido: 22/11/2021 | Revisado: 26/11/2021 | Aceito: 10/12/2021 | Publicado: 17/12/2021

\author{
Amaro Lima Filho \\ ORCID: https://orcid.org/0000-0002-8464-1635 \\ Universidade Federal de Campina Grande, Brasil \\ E-mail: amaroalf@gmail.com \\ Andresa Costa Pereira \\ ORCID: https://orcid.org/0000-0002-3654-6123 \\ Universidade Federal de Campina Grande, Brasil \\ E-mail: andresa@cstr.ufcg.edu.br \\ Marco Antonio Dias da Silva \\ ORCID: https://orcid.org/0000-0002-2774-4769 \\ Universidade Federal de Campina Grande, Brasil \\ E-mail: marco.dias@professor.ufcg.edu.br
}

\begin{abstract}
Resumo
A Pandemia de COVID-19 causou impacto na relação profissional-paciente e na rotina dos graduandos em odontologia. A teleodontologia emergiu apresentando soluções para parte das novas demandas. Contudo, pouco se sabe sobre o ensino de teleodontologia nos cursos de graduação. Este estudo teve como objetivo analisar as matrizes curriculares dos cursos de Odontologia da cidade de São Paulo para buscar informações sobre o ensino de Teleodontologia. Entre agosto e setembro de 2021, utilizando a base de dados oficial do Ministério da Educação (eMEC), foram realizadas buscas, nos sites dos cursos de Odontologia da cidade de São Paulo. Foram tabulados o nome da instituição, presença de site, matriz curricular e/ou PPC disponível, existência da disciplina Telessaúde ou Teleodontologia e o período em que é ministrada. Foram encontrados 21 cursos de odontologia no site do e-MEC, sendo que 18 estão em atividade (89\% privados). A disciplina de Teleodontologia ou Telessaúde só foi encontrada em cinco oportunidades, sendo ofertada em dois dos casos como optativa. Conclui-se que a presença de disciplinas relacionadas à teleodontologia nos cursos da cidade de São Paulo ainda é ínfima, pelo menos no que concerne sua presença nas matrizes curriculares ou projetos pedagógicos dos cursos. Sugere-se a qualificação do corpo docente e que a adequação das matrizes curriculares, decorrente das novas Diretrizes Nacionais dos Cursos de Odontologia, seja utilizada para corrigir a ausência da Teleodontologia na graduação.
\end{abstract}

Palavras-chave: Educação em odontologia; Políticas de saúde; Tecnologia da informação.

\begin{abstract}
The COVID-19 pandemic has caused an impact on both the professional-patient relationship and the routine of undergraduate dental students. Teledentistry arose presenting solutions for part of the new challenges. However, Teledentistry is not mandatory content in the dental curriculum. This study aimed to verify if Telehealth is being taught in Dental courses in the city of Sao Paulo. Between August and September of 2021, using data from the Brazilian Ministry of Education (e-MEC), the dental schools' websites were checked. Information concerning the institution name, website existence, its curriculum, and the presence of a Telehealth or Teledentistry discipline were collected. It was found that 18 , out of 21 dental courses were active ( $89 \%$ private institutions). Only five courses were offering Teledentistry/Telehealth as undergraduate content. In conclusion, the number of dental schools teaching telehealth in São Paulo is limited. Dental schools should capacitate the teaching staff and consider the mandatory curricular changes, due to the new National Guidelines, as a chance to correct the absence of Teledentistry as course content.
\end{abstract}

Keywords: Education, dental; Health policies; Information technology.

\section{Resumen}

La pandemia COVID-19 ha tenido un impacto en la relación profesional-paciente y en la rutina de los estudiantes de odontología. La Teleodontología surgió presentando soluciones para parte de los nuevos desafíos. Todavía, poco se sabe sobre la enseñanza de la teleodontología en los cursos de pregrado. Este estudio tuvo como objetivo verificar el ofrecimiento de la Teleodontología en las matrices curriculares de los cursos de Odontología en la ciudad de São Paulo. Entre agosto y septiembre de 2021, utilizando la base de datos oficial del Ministerio de Educación (e-MEC), se revisaron los sitios web de los cursos de Odontología de la ciudad de São Paulo. Se recopiló información sobre el nombre de la institución, la existencia del sitio web, su plan de estudios y la presencia de una disciplina de Telesalud o Teleodontología y el período en que se imparte. Se encontró que 18 de los 21 cursos de odontología estaban activos (89\% instituciones privadas). Solo cinco cursos ofrecían Teleodontología o Telesalud como contenido curricular. Se 
concluyó que la presencia de disciplinas relacionadas con la Teleodontología en los cursos de la ciudad de São Paulo es muy pequeña, al menos en cuanto a su presencia en el currículo de los cursos. Se sugiere que los profesores sean calificados y que las matrices curriculares sean actualizadas para corregir la ausencia de Teleodontología en la graduación.

Palabras clave: Educación en odontología; Políticas de salud; Tecnología de la información.

\section{Introdução}

A pandemia da COVID-19, decretada em março de 2020 pela Organização Mundial de Saúde (OMS), em decorrência da rápida disseminação do coronavírus, gerou e ainda gera impactos ao nosso cotidiano (Sun et al., 2020; Pereira et al., 2020). A transmissão aérea, a presença do vírus na cavidade bucal e a falta de conhecimento sobre a doença estimularam uma série de medidas como o fechamento de estabelecimentos (Santos et al., 2020). Com os consultórios odontológicos, onde boa parte dos procedimentos gera aerossóis potencialmente contaminados, não foi diferente (Machado et al., 2020).

Esse movimento acelerou a autorização do uso da telemedicina e, a partir disso, passou-se a discutir a implementação da teleodontologia de forma mais geral. De maneira simplificada a teleodontologia apresenta duas vertentes. Uma consiste no uso das tecnologias de informação e comunicação para fornecer possibilidades de educação odontológica e a outra das ações voltadas aos cuidados como teleconsultoria, telediagnóstico, teleinterconsulta etc.

No serviço público de saúde, a teleodontologia já é uma realidade. Não é difícil, também, encontrar bons exemplos do emprego da teleodontologia junto ao Sistema Único de Saúde, como do telessaúde Brasil, única forma autorizada para Pessoa Jurídica no Brasil, de acordo com o Guia de Esclarecimento sobre exercício da Odontologia a distância (CFO, 2020).

Um número limitado de universidades já conta com um núcleo ou um grupo de pesquisa em teleodontologia ou telessaúde, como a Universidade de São Paulo -USP (Núcleo de Teleodontologia e Centro de Produção Digital), a Universidade Estadual do Rio de janeiro - UERJ (Núcleo de Teleodontologia), a Universidade Federal de Santa Catarina UFSC (Serviço de Tele-Estomatologia), a Universidade Federal do rio Grande do Sul - UFRGS (TelessaúdeRS-UFRGS) e a Universidade Federal de campina Grande - UFCG (Teleducação \& Telessaúde), porém pouco se sabe sobre o grau de inserção da Teleodontologia nos cursos de graduação.

Este estudo objetivou analisar as matrizes curriculares dos cursos de Odontologia da cidade de São Paulo para buscar informações sobre o ensino de Teleodontologia.

\section{Metodologia}

Este estudo trata-se de uma pesquisa quantitativa do tipo documental, seguindo a metodologia proposta por Dias da Silva e Pereira (2013), Nobrega et al. (2018) e Medeiros et al. (2020).

Entre 24 de agosto e 08 de setembro de 2021, foi realizado um levantamento de dados sobre os cursos da cidade de São Paulo. A lista completa dos cursos foi obtida da base de dados oficial do Ministério da Educação (e-MEC) de onde também foram coletados dados referentes a categoria administrativa, a situação de atividade do curso e endereço eletrônico de cada uma das Instituições de Ensino Superior (IES). Foram excluídas da amostra os cursos que possuíam situação de atividade "Em extinção" e "Não iniciado".

Após identificadas as IES, os endereços eletrônicos encontrados foram acessados, passou-se a tabulação de informações específicas sobre o curso de Odontologia. No site de cada um dos cursos foram realizadas buscas pela matriz curricular, lista das disciplinas, Projeto Pedagógico do Curso e páginas das disciplinas com o intuito de verificar a existência da oferta de teleodontologia ou telessaúde nos cursos de graduação.

Os cursos que não possuíam matriz curricular disponível no site foram excluídos da amostra. Em seguida os dados foram tabulados e analisados. 


\section{Resultados}

Foram encontrados, com o uso da ferramenta de Consulta Avançada do e-MEC, 21 cursos de odontologia sediados na cidade de São Paulo. Foram encontrados 16 cursos disponibilizando a matriz curricular ou a lista de disciplinas em seu site. Observou-se a existência de disciplina relacionada com a Teleodontologia em apenas cinco oportunidades (Tabela 1).

Tabela 1. Oferta da disciplina de Teleodontologia nos cursos de graduação em Odontologia da cidade de São Paulo.

\begin{tabular}{lccl}
\hline $\begin{array}{l}\text { CATEGORIA } \\
\text { ADMINISTRATIVA }\end{array}$ & $\mathbf{N}^{\circ}$ & $\begin{array}{l}\text { MATRIZ DISPONÍVEL NO } \\
\text { SITE }\end{array}$ & $\begin{array}{l}\text { PRESENÇA DA DISCIPLINA (CARGA } \\
\text { HORÁRIA, SEMESTRE) }\end{array}$ \\
\hline PARTICULAR & 16 & 14 & $3\left(80 \mathrm{~h}, 6^{\mathbf{0}}\right.$ período $)$ \\
\hline PÚBLICA FEDERAL & 0 & & $2(105 \mathrm{~h}$, Optativa $)$ \\
\hline PÚBLICA ESTADUAL & 2 & 2 & \\
\hline TOTAL & 18 & & \\
\hline
\end{tabular}

Fonte: Autores.

\section{Discussão}

Vivemos a revolução social e tecnológica onde quase tudo é conectado e as informações são transmitidas de maneira mais rápida. A Pandemia da COVID-19, que já dura muitos meses, e as medidas para reduzir a disseminação viral têm alterado o comportamento da sociedade e exigido novas alternativas (Schuchmann et al., 2020).

A telessaúde, por exemplo, ajudou a reduzir a circulação de pacientes em ambientes hospitalares, diminuindo o risco de contaminação e melhorando o fluxo dos pacientes nas unidades de saúde (Caetano et al., 2020). Em outros países a teleodontologia foi utilizada para minimizar os problemas gerados pela pandemia (Deshpande et al., 2021).

A Teleodontologia é um ramo da telessaúde, já conhecido no sistema público, que vem crescendo e se aprimorando, cuja importância foi elevada durante a pandemia (Carrer et., 2020). A teleodontologia possibilita a aprendizagem de conteúdo relevante, assim como também incentiva o uso de novas ferramentas tecnológicas durante o período de pandemia (Nascimento et al., 2020). Em atuação com a população, permite redução de custos e maior acesso à opinião de especialistas (Correia et al., 2014). Apesar disso, o presente estudo demonstrou que menos de 30\% dos cursos de Odontologia oferecem a teleodontologia como conteúdo, mesmo que optativo, para a graduação (Tabela 1).

A importância do contato com a teleodontologia durante a graduação ficou ainda mais evidente durante a pandemia, quando seu uso foi efetivado em diversos países e passou a ser discutido no Brasil (Giudice et al., 2020; Fernández et al., 2021; Sousa et al., 2021). A manifestação do Conselho Federal de Odontologia e a necessidade da emissão de um documento posterior para explicar o documento original denotam a complexidade do assunto (CFO, 2020).

A falta de conhecimento em temas emergentes fez com que alunos e cirurgiões dentistas buscassem informações nos meios de comunicação mais utilizados (Connaway et al., 2013). Contudo, pouco se sabe sobre a confiabilidade das fontes e/ou qualidade da informação sobre a Teleodontologia disponível em redes sociais ou plataformas de compartilhamento de conteúdo. O que se sabe ao certo é que o material odontológico encontrado nessas fontes tende a ser considerado de baixa qualidade, pouco confiável e potencialmente danoso (Hegarty et al., 2017; Silva et al., 2019)

O interesse por novos temas também pode gerar um aumento na produção de conteúdo, potencializando o problema da desinformação. Sugere-se, como alternativa a esses problemas, que os cursos de graduação repensem a ausência da teleodontologia na matriz curricular. Concomitantemente, sugere-se que os cursos se dediquem à produção e ao oferecimento de conteúdo de forma gratuita nas mídias digitais visando reduzir os impactos negativos da infodemia (Fernandes et al., 2021). 
Este estudo foi realizado pensando no que ocorre na cidade de São Paulo e apresenta algumas limitações. A falta de informações oficiais e a ausência ou a desatualização de sites impediram a completa análise de todas as instituições. Considerase a amostra consistente, o número de instituições analisadas é maior que o número total de cursos de diversos países da América do Sul e da Europa. Os quatro países do Reino Unido, por exemplo, têm pouco mais de 20 cursos de graduação em Odontologia (General Dental Council, 2018).

\section{Considerações Finais}

Conclui-se que a presença de disciplinas relacionadas à teleodontologia nos cursos da cidade de São Paulo ainda é ínfima, pelo menos no que concerne sua presença nas matrizes curriculares ou projetos pedagógicos dos cursos. Sugere-se que haja qualificação do corpo docente e que a adequação das matrizes curriculares, decorrente das novas Diretrizes Nacionais dos Cursos de Odontologia, seja utilizada para corrigir a ausência da Teleodontologia na graduação. Ademais, é necessário ressaltar a importância da realização de novos estudos para verificar se a pandemia alterou a presença da disciplina teleodontologia nos cursos da cidade de São Paulo, visto que ela ganhou destaque durante o período de isolamento social.

\section{Referências}

Caetano, R., Silva, A. B., Guedes, A. C. C. M., Paiva, C. C. N. D., Ribeiro, G. D. R., Santos, D. L., \& Silva, R. M. D. (2020). Desafios e oportunidades para telessaúde em tempos da pandemia pela COVID-19: uma reflexão sobre os espaços e iniciativas no contexto brasileiro. Cadernos de Saúde Pública, 36, e00088920. https://doi.org/10.1590/0102-311X00088920

Carrer, F. C. D. A., Matuck, B. F., Lucena, E. H. G. D., Martins, F. C., Pucca Junior, G. A., Galante, M. L., Tricoli, M.F.M., Macedo, M.C.S. (2020). Teleodontologia e SUS: uma importante ferramenta para a retomada da Atenção Primária à Saúde no contexto da pandemia de COVID-19. Scielo Preprints. https://preprints.scielo.org/index.php/scielo/preprint/view/837/1159

CFO, Conselho Federal de Odontologia. (2020). Resolução no 226 de 04 de junho de 2020. Dispõe sobre o exercício da Odontologia a distância, mediado por tecnologias, e dá outras providências. http://sistemas.cfo.org.br/visualizar/atos/RESOLUÇÃO/SEC/2020/226

CFO, Conselho Federal de Odontologia. (2020). Guia de Esclarecimento sobre exercício da odontologia a distância. Resolução nº 226 de 04 de junho de 2020. https://website.cfo.org.br/wp-content/uploads/2020/06/Guia-Esclarecimento.pdf

Connaway L, Lanclos D, \& Hood E. "I always stick with the first thing that comes up on Google..." Where People Go for Information, What They Use, and Why. (2013). https://er.educause.edu/articles/2013/12/i-always-stick-with-the-first-thing-that-comes-up-on-google---where-people-go-for-information-whatthey-use-and-why

Correia, A. D. da M. S., Dobashi, B. F., Gonçalves, C. C. M., Monreal, V. R. F. D., Nunes, E. A., Haddad, P. O., \& Sandim, L. V. da S. (2014). Teleodontologia no programa nacional telessaúde Brasil redes: relato da experiência em Mato Grosso Do Sul. Revista Da ABENO, 14(1), 17-29. https://doi.org/10.30979/rev.abeno.v14i1.96

Deshpande, S., Patil, D., Dhokar, A., Bhanushali, P., \& Katge, F. (2021). Teledentistry: A Boon Amidst COVID-19 Lockdown-A Narrative Review. International Journal of Telemedicine and Applications, 2021, 1-5. https://doi.org/10.1155/2021/8859746

Dias da Silva, M. A., \& Pereira, A. C. (2013). Utilização das TIC no ensino complementar da histologia nas faculdades de odontologia do estado de São Paulo. Scientia Plena, 9(10), 1-7.

Fernandes, W. A. M., Nunes, T. K. F., Junior, M. F. S., \& Harmitt, D. D. S. (2021). Infodemia durante a pandemia da covid-19: desafios enfrentados pelos profissionais de saúde na promoção da saúde. Pensar Acadêmico, 19(3), 771-787. https://doi.org/10.21576/pa.2021v19i3.2551

Fernández, C. E., Sigua-Rodriguez, E. A., Vergara-Hernández, C. I., Toral-Rizo, V. H., Tello, G., Madrid, Troconis C. C., Perez Puello, S., \& Roque-Torres, G. D. (2021). Transformação virtual acelerada pela pandemia de COVID-19 na educação odontológica: uma revisão multicêntrica do ensino à distância e da teleodontologia. RGO-Revista Gaúcha de Odontologia.;69, 1-9. https://doi.org/10.1590/1981-86372021002920200171

General Dental Council. Review of Education 2016-2018. https://www.gdc-uk.org/education-cpd/quality-assurance/review-of-education.

Giudice, A., Barone, S., Muraca, D., Averta, F., Diodati, F., Antonelli, A., \& Fortunato, L. (2020). Can Teledentistry Improve the Monitoring of Patients during the Covid-19 Dissemination? A Descriptive Pilot Study. International Journal of Environmental Research and Public Health, 17(10), 3399. https://doi.org/10.3390/ijerph17103399

Hegarty, E., Campbell, C., Grammatopoulos, E., DiBiase, A. T., Sherriff, M., \& Cobourne, M. T. (2017). YouTube ${ }^{\mathrm{TM}}$ as an information resource for orthognathic surgery. Journal of orthodontics, 44(2), 90-96. https://doi.org/10.1080/14653125.2017.1319010

Machado, G. M., Kasper, R. H., Busato, A. L., \& Vinholes, J. I. (2020). Biossegurança e retorno das atividades em odontologia: aspectos relevantes para enfrentamento de covid-19. STOMATOS, 26(50), 3-45. 
Research, Society and Development, v. 10, n. 16, e512101623720, 2021

(CC BY 4.0) | ISSN 2525-3409 | DOI: http://dx.doi.org/10.33448/rsd-v10i16.23720

Medeiros, G. S, Mariano, E. F, \& Silva, M. A. D. (2020). Avaliação do uso do vídeo como ferramenta complementar de ensino nos cursos de saúde da Região Centro-Oeste do Brasil. Research, Society and Development, 9(7), e831974564. http://dx.doi.org/10.33448/rsd-v9i7.4564

Nascimento, A. D. A., Holanda, L. A. L., Borges, B. S., Pereira, F. T., Frade, A. L., Santos, C. B., \& de Vidal, A. K. L. (2020). Teleodontologia, formação profissional e assistência odontológica no sistema único de saúde brasileiro: relato de experiência. Revista Journal of Health, 1(1), 51-67.

Nóbrega, T. E., Ribeiro, E. C., Souto, I. C. C., da Silva, M. A. D., \& Pereira, A. C. (2018). Conteúdo online no ensino odontológico: análise de uma disciplina básica. Journal of Health Informatics, 10(2), 50-4.

Pereira, M. D., Oliveira, L. C. de, Costa, C. F. T., Bezerra, C. M. de O., Pereira, M. D., Santos, C. K. A. dos, \& Dantas, E. H. M. (2020). A pandemia de COVID-19, o isolamento social, consequências na saúde mental e estratégias de enfrentamento: uma revisão integrativa. Research, Society and Development, 9(7), e652974548. https://doi.org/10.33448/rsd-v9i7.4548

Santos, G. M. T., Reis, J. C., Mérida, E. C., Rangel, E. L. F., \& Frich, A. A. (2020). EDUCAÇÃO SUPERIOR: REFLEXÕES A PARTIR DO ADVENTO DA PANDEMIA DA COVID-19. Boletim de Conjuntura (BOCA), 4(10), 108-114. http://dx.doi.org/10.5281/zenodo.4073037

Schuchmann, A. Z., Schnorrenberger, B. L., Chiquetti, M. E., Gaiki, R. S., Raimann, B. W., \& Maeyama, M. A. (2020). Isolamento social vertical X Isolamento social horizontal: os dilemas sanitários e sociais no enfrentamento da pandemia de COVID-19. Brazilian Journal of Health Review, 3(2), 35563576. https://doi.org/10.34119/bjhrv3n2-185

Silva, M. A. D., Pereira, A. C., \& Walmsley, A. D. (2019). Who is providing dental education content via YouTube? British Dental Journal, 226(6), 437-440. https://doi.org/10.1038/s41415-019-0046-8

Sousa, M. A. C., Rego, V. B. J., Lima, N. F. B., Souza, L. D. G., Custódio, L. L. P., Melo, A. T. G., Filho, A. A. O., Figueiredo, C. H. M. C., Ramos, J. C., \& Almeida, M. S. C. (2021). Impacto da Covid-19 no funcionamento das clínicas de radiologia odontológicas no Nordeste do Brasil. Revista Eletrônica Acervo Saúde, 13(3), e6527. https://doi.org/10.25248/reas.e6527.2021

Sun, J., He, W. T., Wang, L., Lai, A., Ji, X., Zhai, X., Li, G., Suchard, M. A., Tian, J., Zhou, J., Veit, M., \& Su, S. (2020). COVID-19: Epidemiology, Evolution, and Cross-Disciplinary Perspectives. Trends in molecular medicine, 26(5), 483-495. https://doi.org/10.1016/j.molmed.2020.02.008 